\title{
Existence and Regularity Results for Harmonic Maps with Potential
}

\author{
Atsushi TACHIKAWA \\ Science University of Tokyo \\ (Communicated by Y. Maeda)
}

\section{Introduction.}

Let $(M, g)$ and $(N, h)$ be Riemannian $m$ - and $n$ - manifolds, $G$ a smooth function on $N$. For a bounded domain $\Omega \subset M$ and a map $u: M \rightarrow N$ we define the energy functional with the potential $G$ on $\Omega$ :

$$
E_{G}(u ; \Omega)=\int_{\Omega}[e(u)-G(u)] d \mu,
$$

where $e(u)$ and $d \mu$ are the standard energy density and the volume element on $M$. Using local coordinate systems $\left(x^{1}, \cdots, x^{m}\right)$ and $\left(u^{1}, \cdots, u^{n}\right)$ on $M$ and $N$ respectively, we can write

$$
E_{G}(u ; \Omega)=\int_{\Omega}\left[\frac{1}{2} g^{\alpha \beta}(x) h_{i j}(u) D_{\alpha} u^{i} D_{\beta} u^{j}-G(u(x))\right] \sqrt{g} d x,
$$

where $\left(g^{\alpha \beta}(x)\right)=\left(g_{\alpha \beta}(x)\right)^{-1}, g=\operatorname{det}\left(g_{\alpha \beta}(x)\right)$ and $D_{\alpha}=\partial / \partial x^{\alpha}$. The Euler-Lagrange equation of $E_{G}$ is given as

$$
\tau(u)+\nabla G=0,
$$

where $\tau(u)$ denotes the tension field of $u$. In local

$$
(\tau(u))^{i}=\frac{1}{\sqrt{g}} D_{\alpha}\left\{\sqrt{g} g^{\alpha \beta} D_{\beta} u^{i}\right\}+g^{\alpha \beta} \Gamma_{j k}^{i} D_{\alpha} u^{j} D_{\beta} u^{k}
$$

A solution $u: \Omega \rightarrow N$ of (1.3) is called to be a harmonic map with potential $G$.

The equations of type (1.3) appear also in some physical contexts. Let $\Omega \subset \mathbf{R}^{m}, N=$ $S^{2}=\left\{(x, y, z) \in \mathbf{R}^{3} ; x^{2}+y^{2}+z^{2}=1\right\}$ and $G(u)=(u, H)=u^{1} H^{1}+\cdots+u^{n} H^{n}$ for some constant vector $H \in \mathbf{R}^{3}$, then the equation (1.3) becomes

$$
\Delta u+u|D u|^{2}-(H, u) u+H=0
$$

Received December 1, 1999

Revised October 12, 2000 
which is called as the static Landau-Lifshitz equation (see [1], [2], [3], [12] and [13]). Here and in the sequel $($,$) and ||$ denote the standard Euclidean inner products and norms respectively. In [2], [12] and [13], Dirichlet problems for the equation (1.4)

$$
\left\{\begin{array}{l}
\Delta u+u|D u|^{2}-(H, u) u+H=0 \\
u=f \text { on } \partial \Omega
\end{array}\right.
$$

are considered.

When $\Omega=B^{3}$, Hong [12] showed the existence of a smooth solution to the Dirichlet problem (1.5), assuming that an extension $u_{0}$ of the boundary value $f$ to $B^{3}$ satisfies

$$
\int_{B^{3}} \frac{1}{2}\left(\left|D u_{0}\right|^{2}+|H| \cdot\left|u_{0}-\frac{H}{|H|}\right|^{2}\right) d x<\varepsilon
$$

for a sufficiently small $\varepsilon>0$.

More recently, Chen [2] showed the existence of a smooth solution to (1.5) for $H=\lambda q$ and $f \in C^{\infty}\left(\partial \Omega, S_{q}^{2}\right)$, where $\lambda$ is a positive constant, $q$ a point in $S^{2}$ and $S_{q}^{2}$ the open hemisphere with the north pole $q$. He also showed the uniqueness of the small solutions i.e. if $u_{1}$ and $u_{2}$ are solutions of the Dirichlet problem with $u_{1}(\Omega), u_{2}(\Omega) \subset S_{q}^{2}$ then $u_{1}=u_{2}$.

On the other hand, when $\Omega=B^{2}$, Hong-Lemaire [13] showed that if $f$ is neither constant $H /|H|$ nor constant $-H /|H|$ then there are at least two different smooth solution to the Dirichlet problem (1.5). Moreover, they showed also that under a certain condition there are at least three different solutions to (1.5).

In this paper, more general cases are treated. We will prove existence of a minimizer of $E_{G}$ in a suitable class of Sobolev maps with the Dirichlet boundary condition

$$
u=f \quad \text { on } \partial \Omega \text {, }
$$

by the direct method of calculus of variations, assuming some conditions on $G, f$ and $N$. We will prove also boundedness and regularity of the minimizer and get existence results for harmonic maps with potential.

Now, let us prepare some notations and terminology. For a Riemannian manifold $N$, $\kappa_{N}(p ; \pi)$ denotes the sectional curvature at $p \in N$ with respect to a plane section $\pi \subset$ $T_{p} N$. Let $q_{0} \in N$ be a fixed point and $I\left(q_{0}\right)$ the injectivity radius of $N$ centered at $q_{0}$. For $p \in B_{I\left(q_{0}\right)}\left(q_{0}\right)$, let $\sigma\left(q_{0}, p\right)(t)$ be the geodesic curve such that $\sigma\left(q_{0}, p\right)(0)=q_{0}$ and $\sigma\left(q_{0}, p\right)(1)=p$. When $\pi$ contains $\sigma^{\prime}\left(q_{0}, p\right)(1)$, let us call $\kappa_{N}(p ; \pi)$ a radial curvature at $p$ with respect to $q_{0}$. We denote as $K_{\mathrm{rad}}\left(p ; q_{0}\right)$ the maximum of radial curvatures of $N$ at $p$ with respect to $q_{0}$, namely

$$
K_{\mathrm{rad}}\left(p ; q_{0}\right)=\max \left\{\kappa_{N}(p ; \pi) \mid \pi \ni \sigma^{\prime}\left(q_{0}, p\right)(1)\right\} .
$$

Throughout this paper we consider the following condition $C\left(q_{0}, R_{0}\right)$ on the radial curvatures of $N$.

$C\left(q_{0}, R_{0}\right)$ : Let $q_{0}$ be a fixed point of $N$ and $R_{0}$ a positive number which is smaller than the injectivity radius $I\left(q_{0}\right)$ of $N$ centered at $q_{0}$. There exists a nonnegative function 
$\rho:\left[0, R_{0}\right) \rightarrow[0, \infty)$ which satisfies the following conditions:

$$
\begin{gathered}
\lim _{t \rightarrow 0} \frac{\rho(t)}{t}=1, \\
\rho(t)>0, \quad \rho^{\prime}(t)>0 \text { for all } t \in\left(0, R_{0}\right), \\
K_{\mathrm{rad}}\left(p ; q_{0}\right) \leq-\frac{\rho^{\prime \prime}}{\rho}\left(\operatorname{dist}\left(q_{0}, p\right)\right) \text { for all } p \in B_{R_{0}}\left(q_{0}\right) .
\end{gathered}
$$

Under this condition, $\exp _{q_{0}}$ gives a normal coordinate system centered at $q_{0}$ on $\boldsymbol{B}_{\boldsymbol{R}_{0}}$. Moreover, with respect to this normal coordinate system we get an estimate on the nonlinear term of $\tau(u)$ which corresponds to the one-sided condition of Giaquinta-Giusti [6]. (See Lemma 2.1.)

EXAMPLES 1. An upper hemisphere $S_{+}^{n}$ satisfies $C\left(q_{0}, R_{0}\right)$ with $q_{0}=$ "the north pole", $R_{0}=\pi / 2$ and $\rho(t)=\sin t$.

2. A simply connected complete manifold $N$ with nonpositive sectional curvatures satisfies $C\left(q_{0}, R_{0}\right)$ with arbitrarily fixed $q_{0} \in N, R_{0}=\infty$ and $\rho(t)=t$.

In section 2 , we will show the existence of minimizers of $E_{G}$ and their $L^{\infty}$-bounds (Theorem 2.2). These lead us to the existence of weak solutions of (1.3).

In section 3, the regularity of the weak solutions whose existence is guaranteed in section 2 will be shown (Theorems 3.2 and 3.3). Thus we will achieve at the existence theorem of harmonic maps with potential (Theorem 3.4).

\section{Existence and global boundedness of a minimizer.}

First of all we prepare an auxiliary geometric lemma.

LEMMA 2.1 (Revised version of [15, Lemma 1.1]). Let $N$ be a Riemannian n-manifold which satisfies $C\left(q_{0}, R_{0}\right),\left(u^{1}, \cdots, u^{n}\right)$ a normal coordinate system centered at $q_{0}$ and $h_{i j}(u)$ the metric tensor with respect to the normal coordinate system. Then we have the following estimates:

$$
h_{i j}(u)\left(X^{i} X^{j}+u^{k} \Gamma_{k l}^{i}(u) X^{j} X^{l}\right) \geq|\zeta|^{2}+t \frac{\rho^{\prime}(t)}{\rho(t)} h_{i j}(y) \xi^{i} \xi^{j}
$$

$$
h_{i j}(u) X^{i} X^{j} \geq|\zeta|^{2}+\frac{\rho^{2}(t)}{t^{2}}|\xi|^{2}
$$

for all $u, X \in \mathbf{R}^{n}$, where $t=|u|, \zeta=t^{-2}(X, u) u$ and $\xi=X-\zeta$.

PROOF. We can proceed as in the proof of [15, Lemma 1.1] or [16, Lemma 2.1], noticing that the assumptions only on the radial curvatures like $C\left(q_{0}, R_{0}\right)$ are sufficient to apply Rauch's comparison theorem. 
REMARK. The estimate (2.1) corresponds to the one-sided condition of GiaquintaGiusti [6] (see the proof of Theorem 3.3).

In the following we assume that the target manifold $N$ satisfies the condition $C\left(q_{0}, R_{0}\right)$ and always use a normal coordinate $\left(u^{1}, \cdots, u^{n}\right)$ centered at $q_{0}$ on $B_{R_{0}}\left(q_{0}\right)$. For some positive constant $R<R_{0}$ and a boundary data $f \in H^{1,2} \cap L^{\infty}\left(\Omega, \mathbf{R}^{n}\right)$ with $\|f\|_{L^{\infty}(\Omega)}<R$ we seek a minimizer of $E_{G}$ in the class

$$
X_{f, R}:=\left\{v \in H^{1,2}\left(\Omega, \mathbf{R}^{n}\right) ; v-f \in H_{0}^{1,2}\left(\Omega, \mathbf{R}^{n}\right),\|v\|_{L^{\infty}(\Omega)} \leq R\right\} .
$$

and show that the minimizer $u$ satisfies the equation (1.3) weakly. (In order to see that minimizer $u$ in the class $X_{f, R}$, we must show the strict inequality $\|u\|_{L^{\infty}}<R$.)

To find a minimizer, we consider the following condition on $G$ :

$$
|G(u)| \leq b_{0}+b_{1}|u|^{\gamma} \text { for some } \gamma \in\left[0,2^{*}\right) \text {. }
$$

Moreover, in order to show the boundedness of $|u|$ we put the following conditions on $\partial G / \partial s(s=|u|)$ :

$$
\left|\frac{\partial G}{\partial s}(u)\right| \leq b_{2}+b_{3}|u|^{\gamma-1} \quad \text { for some } \quad \gamma \in\left[0, \frac{4}{m-2}\right) .
$$

Here, $b_{0}, b_{1}, b_{2}$ and $b_{3}$ are positive constants.

Now, we can state our results on the existence of minimizers of $E_{G}$ in the class $X_{f, R}$ (Theorem 2.2) and $L^{\infty}$-estimate of them (Theorem 2.3).

THEOREM 2.2. Let $(M, g)$ be a smooth Riemannian m-manifold, and $\Omega$ a bounded domain of $M$ with the smooth boundary $\partial \Omega$. Let $(N, h)$ be a smooth Riemannian n-manifold which satisfies the condition $C\left(q_{0}, R_{0}\right)$ for some $q_{0} \in N$ and $R_{0} \in(0,+\infty]$. Assume that $G$ satisfies (2.4). Then for any $R<R_{0}$ and $f \in H^{1,2} \cap L^{\infty}\left(\Omega, \mathbf{R}^{n}\right)$ with $\|f\|_{L^{\infty}(\Omega)}<R$ there exists a minimizer of $E_{G}$ in the class $X_{f, R}$.

Proof. Let $\left\{v_{k}\right\}$ be a minimizing sequence of $E_{G}$ in the class $X_{f, R}$. Since the condition (2.4) implies that

$$
E_{G}(v ; \Omega) \geq c_{0}(g, h) \int_{\Omega}|D v|^{2} d x-c_{1}\left(b_{0}, b_{1}, \gamma, R, \Omega\right)
$$

for every $v \in X_{f, R}$, we see that the sequence $\left\{v_{k}\right\}$ is equibounded in $H^{1,2}$. Therefore, taking subsequence if necessary, we see that

$$
\begin{gathered}
v_{k} \rightarrow u \text { weakly in } H^{1,2}, \\
v_{k} \rightarrow u \text { strongly in } L^{\gamma} \text { for } \gamma \in\left[1,2^{*}\right)
\end{gathered}
$$

for some $u \in\left\{v \in H^{1,2} \cap L^{\gamma}\left(\Omega, \mathbf{R}^{n}\right) ; v-f \in H^{1,2}\right\}$. Here, we used the Kondrachov compactness theorem also. From (2.7) and (2.8), we see easily that

$$
\liminf _{k \rightarrow \infty} E_{G}\left(v_{k} ; \Omega\right) \geq E_{G}(u ; \Omega) .
$$


On the other hand, $L^{\gamma}$-strong convergence implies the almost everywhere convergence, therefore the limit map $u$ belongs to the class $X_{f, R}$. Thus, $u$ minimizes $E_{G}$ in the class $X_{f, R}$.

THEOREM 2.3 Let $(M, g),(N, h), \Omega$ and $f$ be as in Theorem 2.2. Suppose that $2 \leq$ $m \leq 4$ and that $G$ satisfies (2.4) and (2.5). If we have

$$
b_{0}, b_{1}, b_{2}, b_{3}, E_{G}(f),\|f\|_{L^{\infty}(\Omega)}<r_{0}
$$

for a sufficiently small constant $r_{0}>0$, then a minimizer $u$ in the class $X_{f, R}$ satisfies $\|u\|_{L^{\infty}(\Omega)}<R$ for some $R<R_{0}$ and solves (1.3) weakly. Here, $r_{0}$ depends only on $g, h, m$, $\Omega$ and $R_{0}$.

When we can take $R_{0}=+\infty$, the smallness condition (2.9) is not necessary.

PROOF. Let $u$ be a minimizer of $E_{G}$ in the class $X_{f, R}$, then $u$ satisfies

$$
\begin{aligned}
0 & =\left.\frac{d}{d t}\right|_{t=0} E_{G}(u+t \varphi ; \Omega) \\
& =\int_{\Omega}\left[g^{\alpha \beta} h_{i j} D_{\alpha} u^{i}\left\{D_{\beta} \varphi^{j}+\Gamma_{k l}^{j} D_{\beta} u^{l} \varphi^{k}\right\}-\varphi^{i} \frac{\partial G}{\partial u^{i}}\right] \sqrt{g} d x
\end{aligned}
$$

for all $\varphi \in H_{0}^{1,2}\left(\Omega, \mathbf{R}^{n}\right)$ with

$$
u+t \varphi \in X_{f, R} \quad \text { for all } t \quad \text { with }|t|<\varepsilon \quad \text { for some } \varepsilon>0 .
$$

Because of the restriction (2.11), we can not say that (2.10) holds for every test function $\varphi \in C_{0}^{\infty}\left(\Omega, \mathbf{R}^{n}\right)$. Therefore, we can not call $u$ a weak solution yet.

Now, let us show the estimate

$$
\|u\|_{L^{\infty}(\Omega)}<R,
$$

which enables us to call $u$ as a weak solution.

For any nonnegative function $\eta \in C_{0}^{1}(\Omega)$, if we take $\varepsilon>0$ sufficiently small, $u+(-\varepsilon) \eta u$ belongs to the class $X_{f, R}$. Therefore we can take $\varphi=-\varepsilon \eta u$ in (2.10) and get

$$
\begin{aligned}
0= & \int_{\Omega}\left[g^{\alpha \beta}(x) h_{i j}(u)\left\{D_{\alpha} u^{i} D_{\beta} u^{j}+u^{k} \Gamma_{l k}^{j} D_{\alpha} u^{i} D_{\beta} u^{l}\right\} \eta\right. \\
& \left.+g^{\alpha \beta}(x) h_{i j}(u) D_{\alpha} u^{i} u^{j} D_{\beta} \eta-u^{i} \frac{\partial G}{\partial u^{i}} \eta\right] d \mu .
\end{aligned}
$$

Since we are using a normal coordinate on $N,(2.13)$ implies that

$$
\begin{aligned}
& \int_{\Omega} g^{\alpha \beta}(x) h_{i j}(u)\left\{D_{\alpha} u^{i} D_{\beta} u^{j}+u^{k} \Gamma_{l k}^{j} D_{\alpha} u^{i} D_{\beta} u^{l}\right\} \eta d \mu \\
= & -\int_{\Omega}\left\{\frac{1}{2} g^{\alpha \beta}(x) D_{\alpha}|u|^{2} D_{\beta} \eta-u^{i} \frac{\partial G}{\partial u^{i}} \eta\right\} d \mu .
\end{aligned}
$$


On the other hand, from (2.1) of Lemma 2.1, we have

$$
g^{\alpha \beta}(x) h_{i j}(u)\left\{D_{\alpha} u^{i} D_{\beta} u^{j}+u^{k} \Gamma_{l k}^{j} D_{\alpha} u^{i} D_{\beta} u^{l}\right\} \geq \delta|D u|^{2}
$$

for

$$
\delta=\min \left\{1, \inf _{0<t<R} t \rho^{\prime}(t) / \rho(t)\right\}>0 .
$$

Thus, under the condition $C\left(q_{0}, R_{0}\right)$, we get for all $\eta \in C_{0}^{1}(\Omega)$ with $\eta \geq 0$ that

$$
0 \geq \int_{\Omega}\left\{\frac{1}{2} g^{\alpha \beta}(x) D_{\alpha}|u|^{2} D_{\beta} \eta-|u| \frac{\partial G}{\partial s} \eta\right\} d \mu,
$$

where $s=|u|=\operatorname{dist}\left(q_{0}, u(x)\right)$. Since $u$ belongs to the class $X_{f, R}, u$ is essentially bounded and therefore $D|u|^{2}$ is in the class $L^{2}$, namely $|u|^{2} \in H^{1,2}$.

Let $w=|u|^{2}-|f|^{2}$, then from (2.16), $w$ satisfies

$$
\int_{\Omega}\left\{g^{\alpha \beta}(x) D_{\alpha} w D_{\beta} \eta-g^{\alpha \beta}(x) D_{\alpha}|f|^{2} D_{\beta} \eta+|u| \frac{\partial G}{\partial s} \eta\right\} d \mu \leq 0
$$

for any $\eta \in C_{0}^{1}(\Omega)$ with $\eta \geq 0$. Assume that $|u| \partial G / \partial s \in L^{q}(\Omega)$ for some $q>m / 2$, then using [9, Theorem 8.15] we get

$$
\sup _{\Omega}|w| \leq c_{2}(m, g, \Omega)\left(\|u\|_{L^{4}}+\|f\|_{L^{2 q}}+\left\||u| \frac{\partial G}{\partial s}\right\|_{L^{q}}\right) .
$$

Now, let us estimate the right hand side of (2.18). Since we are assuming (2.4), the minimality of $u$ implies that

$$
\begin{aligned}
\int_{\Omega} e(u) d \mu & \leq \int_{\Omega} G(u) d \mu+E_{G}(f) \\
& \leq E_{G}(f)+b_{0} \operatorname{vol} .(\Omega)+b_{1} \int_{\Omega}|u|^{\gamma} d \mu \\
& \leq E_{G}(f)+b_{0} \operatorname{vol} .(\Omega)+b_{1} \int_{\Omega}\left\{\varepsilon|u|^{2^{*}}+\varepsilon^{-\frac{\gamma}{2^{*}-\gamma}}\right\} d \mu \\
& \leq c_{3}\left(E_{G}(f), \Omega, g, \varepsilon, \gamma, b_{0}, b_{1}\right)+\varepsilon c_{4}\left(\Omega, g, h, b_{1}\right) \int_{\Omega} e(u) d \mu .
\end{aligned}
$$

Here, we used Young's inequality and the Sobolev inequality. By choosing $\varepsilon>0$ sufficiently small, we get the following a-priori estimate:

$$
\int_{\Omega}|D u|^{2} d x \leq c_{5}\left(g, h, \gamma, b_{0}, b_{1}, \Omega, E_{G}(f)\right) .
$$

Using the Sobolev inequality and the assumption that $2 \leq m \leq 4$, from (2.19) we get

$$
\|u\|_{L^{4}} \leq c_{6}(\Omega, m)\|u\|_{L^{2^{*}}} \leq c_{6} K_{0}\left(g, h, \gamma, b_{0}, b_{1}, \Omega, E_{G}(f)\right) .
$$

for some positive constants $c_{6}$ and $K_{0}$. Here, it is nothing to see that $K_{0}$ satisfies

$$
\lim _{b_{0}, b_{1}, E_{G}(f) \rightarrow 0} K_{0}\left(g, h, \gamma, b_{0}, b_{1}, \Omega, E_{G}(f)\right)=0 .
$$


On the other hand, using the condition (2.5), we see that

$$
\left\||u| \frac{\partial G}{\partial s}\right\|_{L^{q}} \leq c_{7}\left(b_{2}, b_{3}, q, \Omega\right)\|u\|_{L^{2 *}} \text { for } q=\min \left\{2^{*}, 2^{*} / \gamma\right\}>m / 2 .
$$

Thus, if $2 \leq m \leq 4$ and (2.5) holds, we obtain from (2.18)

$$
\sup _{\Omega}|u|^{2} \leq c_{4}\left\{c_{6}\left(1+c_{7}\right) K_{0}+\|f\|_{L^{2 q}}\right\}+\sup _{\Omega}|f|^{2} .
$$

Now, from (2.21) and (2.22), we can see that if $b_{0}, b_{1}, b_{2}, b_{3}, E_{G}(f)$ and $\|f\|_{L^{\infty}(\Omega)}$ are sufficiently small we have (2.12).

When we can take $R_{0}=+\infty$, for any given $b_{0}, b_{1}, b_{2}, b_{3}$ and $f$ we can choose $R$ sufficiently large so that $R$ is greater than the right hand side of (2.22).

\section{Regularity of minimizers.}

In this section we show the $C^{0, \alpha}$-regularity of a minimizer $u$ under the condition (2.5).

When the boundedness of a minimizer $u$ of $E_{G}$ is given, we can easily see that the results of [6] and [14] are valid for our case. More precisely we have the following theorems:

THEOREM 3.1. Let $M, N, \Omega$ and $f$ be as in Theorem 2.2 and $G$ a smooth function defined on $N$. Assume that $u$ minimize $E_{G}$ in the class $X_{f, R}$ and that $\|u\|_{L^{\infty}(\Omega)}<R$. Then there exists an open set $\Omega_{0} \subset \Omega$ such that $u \in C^{0, \alpha}\left(\Omega_{0}, \mathbf{R}^{n}\right)$ for every $\alpha \in(0,1)$. Moreover,

$$
\Omega \backslash \Omega_{0}=\left\{x_{0} \in \Omega ; \liminf _{r \rightarrow 0} r^{2-n} \int_{B_{r}\left(x_{0}\right)}|D u|^{2} d x>\varepsilon_{0}\right\}
$$

where $\varepsilon_{0}$ is a positive constant independent of $u$. Finally

$$
\mathcal{H}^{n-q}\left(\Omega \backslash \Omega_{0}\right)=0
$$

for some $q>2, \mathcal{H}^{n-q}$ denoting $(n-q)$-dimensional Hausdorff measure.

PROOF. It is enough to proceed as the proof of [6, Theorem 5.1], adding $\int G(u) d x$ to their quadratic functional. We will get

$$
\begin{aligned}
& \int_{B \rho}\left(1+|D u|^{2}\right) d x \\
\leq & c_{8}\left[\left(\frac{\rho}{r}\right)^{m}+\omega\left(r^{2}+c_{9} r^{2-m} \int_{B_{r}}|D u|^{2} d x\right)^{1-2 / q}\right] \int_{B_{2 r}}|D u|^{2} d x+c_{10} r^{m},
\end{aligned}
$$

instead of $[6,(5.11)]$. Now, the assertion follows from the above estimate using "a useful lemma" on $[5$, p. 44].

THEOREM 3.2. Let $M, N, \Omega, G$, fand $u$ be as in Theorem 3.1. Assume that the boundary data $f$ is in the class $H^{1, s}(\Omega, N)$ for some $s>m$. Then $u$ is Hölder continuous in a neighborhood of $\partial \Omega$. 
PROOF. Let $x_{0}$ be an arbitrary point on $\partial \Omega$ and choose a local coordinate system so that $x_{0}=0$. As in [14], let us consider the blown-up functions

$$
u_{(\nu)}(x)=u(x / v), \quad g_{(\nu)}^{\alpha \beta}(x)=g^{\alpha \beta}(x / v), \quad(v=1,2,3, \ldots) .
$$

Then $u_{(v)}$ minimizes the functional

$$
\int\left[\frac{1}{2} g_{(v)}^{\alpha \beta}(x) h_{i j}(v) D_{\alpha} v^{i} D_{\beta} v^{j}-\frac{1}{v^{2}} G(v(x))\right] \sqrt{g_{(v)}} d x,
$$

and converges to a minimizer $v$ of the functional

$$
\int\left[\frac{1}{2} g^{\alpha \beta}(0) h_{i j}(v) D_{\alpha} v^{i} D_{\beta} v^{j}\right] \sqrt{g} d x
$$

Namely, the potential term disappears in the blowing-up process. Thus, we can proceed as in [14] and get the assertion.

Now, we prove the following regularity theorem for minimizers of $E_{G}$.

THEOREM 3.3. Suppose that all assumptions in Theorem 3.2 are satisfied. Let $u$ be a minimizer of $E_{G}$ in the class $X_{f, R}$ which satisfies $\|u\|_{L^{\infty}(\Omega)}<R$. Then $u$ is Hölder continuous on $\bar{\Omega}$.

PROOF. By virtue of Theorems 3.1 and 3.2, it is enough to show that for every $\varepsilon_{0}>0$ and $x \in \Omega$ there exists a positive constant $\rho>0$ such that

$$
\rho^{2-n} \int_{B_{\rho}(x)}|D u|^{2} d x \leq \varepsilon_{0}
$$

To show (3.2) we can proceed similarly as in [6] by remarking that the estimate (2.15) plays the role of the one-sided condition of [6].

Let $x \in \Omega$ be an arbitrarily fixed point and $r$ a positive constant such that $B_{2 r}(x) \subset \subset \Omega$. Choosing $\eta \geq 0$ in (2.14) so that spt $\eta \subset B_{2 r}(x)$ and using (2.15) we get

$$
\delta \int_{B_{2 r}(x)}|D u|^{2} \eta d \mu \leq-\int_{B_{2 r(x)}}\left\{\frac{1}{2} g^{\alpha \beta}(x) D_{\alpha}|u|^{2} D_{\beta} \eta-|u| \frac{\partial G}{\partial s}\right\} d \mu .
$$

Since we are assuming that $\|u\|_{L^{\infty}}<R$, we have

$$
|| u\left|\frac{\partial G}{\partial s}\right| \leq K
$$

for some positive constant $K$ which depends only $g, h, G$ and $f$. Let $M(r)=\sup _{B_{r}(x)}|u|$ and $z=M^{2}(2 r)-|u|^{2}$. Then from (3.3) and (3.4) we get

$$
0 \leq \int_{B_{r}(x)}\left(g^{\alpha \beta}(x) D_{\alpha} z D_{\beta} \eta+K\right) d \mu .
$$

Thus, $z$ is a nonnegative supersolution of a uniformly elliptic equation and therefore, using the weak Harnack inequality (see [9, Theorem 8.18]), we obtain

$$
r^{-m} \int_{B_{2 r}(x)}|z| d x \leq c_{11}(g, m, K)\left(\inf _{B_{r}(x)} z+r^{2}\right) .
$$


Let $w \in C^{2}\left(B_{2 r}(x)\right) \cup C\left(B_{2 \bar{r}}(x)\right)$ be a solution of the Dirichlet problem

$$
\left\{\begin{aligned}
D_{\beta}\left\{\sqrt{g} g^{\alpha \beta}(x) D_{\alpha} w\right\} & =-\frac{1}{r^{2}} \text { in } B_{2 r}(x), \\
w & =0 \text { on } \partial B_{2 r}(x) .
\end{aligned}\right.
$$

Then, $w$ is bounded from above by a positive constant $\alpha_{1}$ in $B_{2 r}(x)$ (see for example [9, Theorem 3.7]). On the other hand, since the right hand side of the above equation is negative, $w$ is a positive supersolution of the equation $D_{\beta}\left\{\sqrt{g} g^{\alpha \beta}(x) D_{\alpha} w\right\}=0$, and therefore by the weak Harnack inequality we have that $w \geq \alpha_{2}$ in $B_{r}(x)$ for some positive constant $\alpha_{2}$. Here, $\alpha_{1}$ and $\alpha_{2}$ does not depend on $r$. Indeed, if $w_{1}$ is a solution of the above Dirichlet problem for $r=1$, then $w(x)=w_{1}(t x)$ solves the Dirichlet problem for $r=t$.

Now, $w$ is in the class $H_{0}^{1,2}\left(B_{2 r}(x)\right)$ clearly and satisfies the following weak from of the equation

$$
\int_{B_{2 r}(x)} g^{\alpha \beta}(x) D_{\beta} w D_{\alpha} \varphi \sqrt{g} d x=r^{-2} \int_{B_{2 r}(x)} \varphi d x \text { for all } \varphi \in H_{0}^{1,2}\left(B_{2 r}(x)\right) .
$$

Let $\varphi=w z$ in (3.7), then we have

$$
\frac{1}{2} \int_{B_{2 r}(x)} g^{\alpha \beta} D_{\beta} w^{2} D_{\alpha} z d \mu+\int_{B_{2 r}(x)} g^{\alpha \beta} D_{\beta} w D_{\alpha} w z d \mu=r^{-2} \int_{B_{2 r}(x)} w z d x,
$$

and therefore

$$
\frac{1}{2} \int_{B_{2 r}(x)} g^{\alpha \beta} D_{\beta} w^{2} D_{\alpha} z d x \leq \alpha_{1} r^{-2} \int_{B_{2 r}(x)} z d x .
$$

Since $w^{2}$ is in the class $H_{0}^{1,2}$ also, we can take $\eta=w^{2}$ in (3.3). Taking $\eta=w^{2}$ in (3.3) and using (3.6) and (3.8), we get

$$
\begin{aligned}
\delta \alpha_{2}^{2} \int_{B_{r}(x)}|D u|^{2} d \mu & \leq \frac{1}{2} \int_{B_{2 r}(x)} g^{\alpha \beta} D_{\alpha} z D_{\beta} \eta d \mu+\int_{B_{2 r}(x)} K w^{2} d \mu \\
& \leq \alpha_{1} r^{-2} \int_{B_{2 r}(x)} z d x+K \alpha_{1}^{2}(2 r)^{m} \\
& \leq \alpha_{1} c_{12} r^{m-2} \inf _{B_{r}(x)} z+c_{13} r^{m}
\end{aligned}
$$

Thus we obtain

$$
r^{2-m} \int_{B_{r}(x)}|D u|^{2} d x \leq c_{14}\left\{\inf _{B_{r}(x)} z+r^{2}\right\} \leq c_{14}\left\{M^{2}(2 r)-M^{2}(r)+r^{2}\right\} .
$$

On the other hand $u$ is bounded and therefore

$$
\sum_{k=0}^{+\infty}\left[M^{2}\left(2^{1-k} r\right)-M^{2}\left(2^{k} r\right)\right] \leq M^{2}(2 r) \leq \sup _{\Omega}|u|^{2} .
$$

Thus (3.10) implies (3.2) with $\rho=2^{-k} r$ for some $k$.

Now, combining Theorems 2.2, 3.3 and the standard Schauder estimates, we get the following existence theorem. 
THEOREM 3.4. Let $M, N$ and $\Omega$ be as in Theorem 2.2 and $r_{0}$ as in Theorem 2.3. Suppose that a smooth function $G(u)$ satisfies (2.4) and (2.5) with $b_{i}<r_{0}(i=0,1,2,3)$ and that the boundary data $f$ is in the class $H^{1, s} \cap L^{\infty}\left(\Omega, \mathbf{R}^{n}\right)$ for some $s>m$ and satisfies $E_{G}(f),\|f\|_{L^{\infty}(\Omega)}<r_{0}$. Then there exists a minimizer $u$ of $E_{G}$ in the class $X_{f, R}$ for some $R<R_{0}$. Moreover, the minimizer $u$ is in the class $C^{2, \alpha}\left(\Omega, B_{R}\right) \cap C^{0, \alpha}\left(\bar{\Omega}, B_{R}\right)$ and a harmonic map with potential $G$.

If we can take $R_{0}=\infty$, the smallness conditions on $b_{i}(i=0,1,2,3), E_{G}(f)$ and on $\|f\|_{L^{\infty}(\Omega)}$ are not necessary.

\section{References}

[ 1] Q. CHEN, Liouville theorem for harmonic maps with potential, Manuscripta Math. 95 (1998), 507-517.

[2] Q. CHEN, Maximum principle, uniqueness and existence for harmonic maps with potential and LandauLifshitz equations, Calc. Var. 8 (1999), 91-107.

[ 3 ] A. FARDoUn and A. RATtO, Harmonic maps with potential, Calc. Var. 5 (1997), 183-197.

[4] M. GIAQuinta, Multiple Integrals in the Calculus of Variations and Non-linear Elliptic Systems, Ann. of Math. Stud. 105 (1983), Princeton Univ. Press.

[ 5 ] M. GIAQUINTA, Introduction to regularity theory for nonlinear elliptic equations, Lectures in Mathematics ETH Zürich, Birkhäuser (1993).

[ 6 ] M. Giaquinta and E. GiUsti, On the regularity of the minima of variational integrals, Acta Math. 148 (1982), 31-46.

[ 7 ] M. GIQUINTA and E. GiUSTI, Differentiability of minima of non-differentiable functionals, Invent. Math. 72 (1983), 285-298.

[ 8 ] D. Gromoll, W. Klingenberg and W. Meyer, Rimannsche Geometrie im Großen, Lecture Notes in Math. 55 (1968), Springer.

[ 9 ] D. GILBERG and N.S. TRUDINGER, Elliptic partial differential equations of second order (2nd ed. revised 3rd printing), Springer (1998).

[10] S. HildebRANDT, J. JosT and K.-O. WidMAN, Harmonic mappings and minimal submanifolds, Invent. Math. 62 (1980), 269-298.

[11] S. HildebRANDT and H. KAUL, Two-Dimensional variational problems with obstructions and Plateau's problem for H-surfaces in a Riemannian manifold, Comm. Pure Appl. Math. 25 (1972), 187-223.

[12] M.-C. HoNG, The Landau-Lifshitz equation with the external field-a new extension for harmonic maps with values in $S^{2}$, Math. Z. 220 (1995), 171-188.

[13] M.-C. Hong and L. Lemaire, Multiple solutions of the static Landau-Lifshitz equation from $B^{2}$ into $S^{2}$, Math. Z. 220 (1995), 295-306.

[14] J. Jost and M. MEIER, Boundary regurality for minima of certain quadratic functionals, Math. Ann. 262 (1983), 549-561.

[15] A. TACHIKAWA, On interior regularity and Liouville's theorem for harmonic mappings, Manuscripta Math. 42 (1983), 11-40.

[16] A. TACHIKAWA, Harmonic mappings from $\mathbf{R}^{m}$ into an Hadamard manifold, J. Math. Soc. Japan 42 (1990), 147-153.

[17] A. TACHIKAWA, Harmonic maps from a Riemannian manifold with a pole into an Hadamard manifold with negative sectional curvatures, Manuscripta Math. 74 (1992), 69-81.

Present Address:

DEPARTMENT OF MATHEMATICS, FACULTY OF SCIENCE AND TECHNOLOGY, SCIENCE UNIVERSITY OF TOKYO, NODA, CHIBA, 278-85 10 JAPAN.

e-mail: atsushi@ma.noda.sut.ac.jp 